\title{
Enteógenos e Inteligência Existencial: Plantas Mestres como Instrumentos Cognitivos ${ }^{1}$
}

\author{
Kenneth W. Tupper ${ }^{2}$
}

\begin{abstract}
Resumo:
A partir de recentes mudanças nas legislações em relação às drogas em diferentes países, investiguei o potencial dos enteógenos (i.e., plantas psicoativas usadas como sacramentos espirituais) como instrumentos para favorecer a inteligência existencial. Plantas Mestres das Américas como a ayahuasca, os cogumelos de psilocibina, o peiote e o soma indo-ariano da Eurásia, são alguns dos exemplos de enteógenos que foram e continuam a ser utilizados, no passado e no presente. Todas estas plantas foram reverenciadas por pessoas e culturas diferentes como instrumentos cognitivos que promovem um mais rico entendimento cosmológico do mundo. Utilizo a teoria das múltiplas inteligências revisada por Gardner (1999) e sua formulação de uma inteligência "existencial" como uma lente teórica através da qual se deve considerar as possibilidades cognitivas dos enteógenos e explorar potenciais caminhos na Educação.
\end{abstract}

Palavras chaves: múltiplas inteligências; inteligência existencial; enteógeno, ayahuasca; alucinógenos.

\section{Abstract:}

In light of recent specific liberalizations in drug laws in some countries, I have investigated the potential of entheogens (i.e., psychoactive plants used as spiritual sacraments) as tools to facilitate existential intelligence. "Plant teachers" from the Americas such as ayahuasca, psilocybin mushrooms, and peyote, and the Indo-Aryan soma of Eurasia, are examples of entheogens that have been used in both the past and present. These have all been revered as spiritual or cognitive tools to provide a richer cosmological understanding of the world for both individuals and cultures. I used Gardner's (1999a) revised multiple intelligence theory and his postulation of an "existential" intelligence as a theoretical lens through which to

\footnotetext{
1 Traduzido por Mauro Sá Rego Costa de Tupper, K.W. (2002). Entheogens and existential intelligence: The use of plant teachers as cognitive tools. Canadian Journal of Education. 27(4),499-516. http://www.csse-scee.ca/CJE/Articles/FullText/CJE27-4/CJE27-4-tupper.pdf), com a autorização do Canadian Journal of Education.

2 Kenneth W. Tupper, Ph.D. é Adjunct Professor, School of Population \& Public Health. University of British Columbia. www.kentupper.com
} 
account for the cognitive possibilities of entheogens and explore potential ramifications for education.

Keywords:multiple intelligences; existential intelligence; entheogen; ayahuasca; hallucinogen.

Neste artigo, utilizo e desenvolvo os potenciais do conceito de inteligência existencial como formulado por Gardner (1999a). Além disso, coloco a possibilidade de que algumas espécies de substâncias psicoativas - enteógenos - tenham o potencial de favorecer este tipo de inteligência. A questão surge a partir da recente liberalização em legislações sobre drogas em diversos países do ocidente, permitindo o uso sacramental da ayahuasca, um chá psicoativo preparado com plantas originais da Amazônia. Desafio os leitores a desfazer-se do antigo paradigma dominante na cultura ocidental moderna que encarava as drogas alucinógenas, a priori, como necessariamente maléficas e desprovidas de valor. O objetivo de minha discussão é confrontar suposições sobre as drogas que injustamente perpetuavam a depreciação e proibição do uso de alguns tipos de substâncias psicoativas. Mais amplamente, questiono suposições sobre a inteligência que limitam o escopo do pensamento educacional contemporâneo.

Alguns pesquisadores criaram 0 termo enteógeno para substituir o termo psicodélico (Ruck, Bigwood, Staples, Ott, \& Wasson, 1979) que carregava conotações pesadas associadas aos paradigmas clínicos e sócio-culturais trazidos de experiências dos anos 60 e assim designar apropriadamente as plantas e substâncias substâncias reverenciadas em rituais tradicionais. Utilizo ambos os termos neste artigo: enteógeno quando me refiro à substância usada como um instrumento espiritual e sacramental, e psicodélico quando me refiro a inúmeros propósitos durante ou seguindo a assim chamada era psicodélica dos anos 60 (reconhecendo que alguns usos contemporâneos não-indígenas possam ser enteogênicos - as categorias não são, com certeza, claramente distintas). Que tipos de 
plantas ou substâncias químicas caem na categoria de enteógenos é uma questão, na medida em que diferentes culturas em épocas diferentes veneraram um grande número de inebriantes - da coca à cannabis ao álcool e o ópio - como presentes dos deuses (ou de Deus). No escopo deste artigo, no entanto, focalizo a classe de drogas que Lewin (1924/1997) denominou como phantastica - nome derivado da palavra grega para a faculdade da imaginação (Shorter Oxford English Dictionary, 1973). Mais tarde, estas substâncias tornaram-se conhecidas como alucinógenos, classe que inclui o ácido lisérgico e substâncias associadas como a psilocibina, a mescalina e a dimethyltryptamina. Com exceção da mescalina, todas compartilham estruturas químicas similares; todos, incluindo a mescalina, produzem efeitos fenomenológicos semelhantes; e, mais importante para a discussão atual, todos têm uma história de uso ritual como substâncias psicoespirituais ou, como formulo, instrumentos culturais para facilitar o conhecimento (Schultes \& Hofmann, 1992).

A questão do uso de enteógenos na cultura ocidental moderna torna-se mais significativa à luz de alguns precedentes legais em países como o Brasil, a Holanda, a Espanha, e brevemente, talvez, os Estados Unidos e o Canadá. A ayahuasca, que discuto, com mais detalhe na próxima seção sobre as plantas mestres, foi legalizada para uso religioso por povos não-indígenas, no Brasil, em $1987 .{ }^{3}$ Um grupo brasileiro, o Santo Daime, a estava utilizando como sacramento em cerimônias na Holanda, quando, no outono de 1999, autoridades intervieram e prenderam seus líderes - o primeiro caso de intolerância religiosa pelo governo holandês nos últimos 300 anos. Uma ação legal posterior, baseada nas leis de direitos humanos da União Europeia, liberou-os de todas as acusações, estabelecendo um precedente para o resto da Europa (Adelaars, 2001). Um caso similar na Espanha resultou na permissão do governo espanhol para o direito de uso da ayahuasca no país. Uma recente decisão judicial 
nos Estados Unidos, pela 10th Circuit Court of Appeals, em 4 de setembro de 2003, decidiu a favor da liberdade religiosa do uso da ayahuasca (Center for Cognitive Liberty and Ethics, 2003). E no Canadá, um processo junto ao Health Canada e ao Departmento de Justiça, pela suspensão do Controlled Drugs and Substances Act está em curso e deverá permitir à Igreja do Santo Daime o uso religioso de seu sacramento, conhecido como Daime ou Santo Daime (J. W. Rochester, comunicação pessoal, 8 de Outubro de 2003). ${ }^{4}$

Uma questão levantada por essa tendência de liberalização em regimes regulatórios normalmente proibicionistas beneficia substâncias como a ayahuasca. A discussão a seguir toma esta questão na sua relação com teorias psicológicas contemporâneas sobre a inteligência e leva a articulações potenciais para a educação. A próxima sessão examina a metáfora das plantas mestras, que não é rara entre culturas que praticaram tradicionalmente com o uso de plantas enteogênicas. Em seguida, uso a teoria das múltiplas inteligências de Gardner (1983) como um enquadramento teórico para dar conta das implicações cognitivas do uso dos enteógenos. E para terminar, retomo a discussão da possível relevância da inteligência existencial e dos enteógenos na educação.

\section{Plantas Mestras}

Antes de seguir para uma discussão mais ampla da(s) inteligência(s), apresento informações introdutórias sobre a ayahuasca e os enteógenos. A ayahuasca foi reverenciada como planta mestra entre dezenas de povos indígenas sul-americanos por muitos séculos (Luna, 1984; Schulles \& Hoffmann, 1992). A palavra ayahuasca, da língua Quechua, de povos indígenas do Equador e Peru, traduz-se como "vinha da alma" (Metzner, 1999). Refere-se a um chá feito de um cipó silvestre, o Banisteriopsis caapi, misturado a outras plantas da família do café, Psychotria viridis (McKenna, 1999). Estas duas plantas contem, respectivamente alcaloides de harmala e 
dimethyltryptamina, duas substânciasque quando ingeridas oralmente criam uma sinergia bioquímica capaz de produzir profundas alterações de consciência (Grob, et al., 1996; McKenna, Towers,\& Abbot, 1984). Entre os povos indígenas da Amazônia, a ayahuasca é uma das mais valorizadas plantas medicinais e sacramentais de sua farmacopeia. Embora os xamãs em tribos diferentes usem o chá para propósitos variados e tenham diferentes receitas para ele, a aplicação da ayahuasca como instrumento eficaz para se atingir entendimento e sabedoria é comum a todos (Brown, 1986; Dobkin de Rios, 1984).

Apesar da explosão do interesse popular pelas drogas psicoativas durante os anos 60 , a ayahuasca manteve-se até muito recentemente desconhecida da cultura ocidental. ${ }^{5}$ No entanto, o final do século XX viu o crescimento de movimentos religiosos entre povos não-indígenas no Brasil, sincretizando o uso da ayahuasca com o simbolismo cristão, o espiritualismo africano, e rituais nativos. Duas das mais expandidas igrejas ayahuasqueiras são o Santo Daime (Santo Daime, 2004) e a União do Vegetal (União do Vegetal, 2004). Estas organizações, nas últimas décadas, ganharam legitimação como válidas e mesmo valiosas práticas espirituais, promovendo benefícios sociais, psicológicos e espirituais (Grob, 1999; Riba, et al., 2001).

A ayahuasca não é a única planta mestra no panteão dos instrumentos enteogênicos. Outros povos indígenas das Américas utilizaram os cogumelos da psilocibina durante milênios com propósitos espirituais e de cura (Dobkin de Rios, 1973; Wasson, 1980). De modo similar, o cacto do peyote, com uma longa história de uso entre grupos indígenas mexicanos (Fikes, 1996; Myerhoff, 1974; Stewart,1987), é utilizado amplamente nos Estados Unidos pela Native American Church (LaBarre, 1989; Smith \& Snake, 1996). E mesmo na história mais antiga da cultura do Ocidente, os textos 
indo-arianos do Rig Veda cantam loas ao divinizado soma (Pande, 1984). Embora a identidade taxonômica do soma tenha se perdido, ele parece ter sido uma planta ou cogumelo com o poder de induzir com segurança experiências místicas (Eliade, 1978; Wasson, 1968). A variedade dos enteógenos estende-se, certamente, bem além dos exemplos que trouxe aqui. No entanto, a ayahuasca, os cogumelos da psilocibina, o peyote e o soma são exemplares de plantas que foram culturalmente valorizados por seus efeitos psicológicos e espirituais sobre indivíduos e comunidades.

Neste trabalho, defendo que a importância dos enteógenos está no seu papel como instrumentos, como mediadores entre a mente e o ambiente. Definir uma droga psicoativa como um instrumento talvez um conceito novo para alguns - invoca sua capacidade de efetuar uma mudança pretendida na mente/corpo. Comentando as noções de Vigotsky de instrumentos psicológicos, John-Steiner e Souberman (1978) observam que "o uso de um instrumento tem.... importantes efeitos sobre relações internas e funcionais no interior do cérebro humano" (p. 133). Embora eles não estivessem certamente pensando em drogas como instrumentos, o significado desta observação torna-se ainda mais literal quando os instrumentos em questão são plantas ou substânciasquímicas ingeridas com o objetivo de afetar a consciência pela manipulação da química cerebral. Na verdade, plantas ou substâncias químicas psicoativas parecem desafiar a divisão tradicional entre instrumentos físicos ou psicológicos, que afetem a mente/corpo (entendidas pelos psicólogos modernos como inseparáveis). É importante considerar o potencial dos enteógenos não só por seus efeitos neuropsicológicos imediatos, mas também pelas práticas sociais - rituais - nas quais seu uso foi incorporado pelas tradições (Dobkin de Rios, 1996; Smith, 2000). O valor de proteção que o ritual provê ao uso do enteógeno é evidente, quando se considera o envolvimento universal das práticas rituais. (Weil, 1972/1986). Evidência médica sugere que há riscos fisiológicos 
mínimos associados às drogas psicodélicas (Callaway, et al., 1999; Grinspoon \& Bakalar, 1979/1998; Julien, 1998). Hofmann (1980), o químico que primeiro sintetizou acidentalmente a dietilamida do ácido lisérgico (LSD), coloca que os riscos psicológicos associados aos psicodélicos na cultura ocidental moderna são função de seu uso recreativo em circunstâncias pouco seguras. Um contexto ritual, no entanto, oferece as salvaguardas psicoespirituais que fazem do potencial das plantas mestras enteogênicas ampliarem conhecimentos uma intrigante possibilidade.

\section{Inteligência Existencial}

Gardner (1983) desenvolveu uma teoria das múltiplas inteligências que postulava originalmente sete tipos de inteligência. ${ }^{6}$ Desde então, ele acrescentou uma inteligência naturalista e sugeriu a possibilidade de uma inteligência espiritual (1999a ; 1999b). Não desejando se aprofundar muito em território repleto de armadilhas teológicas, Gardner (1999a) preferiu a denominação de inteligência existencial no lugar da inteligência espiritual (p. 123). A inteligência existencial, como Gardner a caracteriza, envolve uma capacidade ampliada de apreciar e considerar os enigmas cosmológicos que definem a condição humana, uma consciência excepcional dos mistérios ontológicos, metafísicos e epistemológicos quetêm sido uma preocupação perene para povos de todas as culturas.

$\mathrm{Na}$ formulação original de sua teoria, Gardner (1999a) desafiava as (estreitas) definições correntes da inteligência com uma mais ampla que via a inteligência como "a habilidade de solucionar problemas ou definir produtos que sejam valorizados por ao menos uma cultura ou comunidade" (p. 113). Ele propôs oito critérios, ou índices, para identificar uma inteligência; no entanto, observou que estes não constituem condições necessárias para determinar uma inteligência, mas simples desiderata que uma candidata a inteligência 
deveria demonstrar (1983, p.62). Ele admitiu ainda que nenhuma das suas sete inteligências originais preenchia todos os critérios, embora todas respondessem a pelo menos oito destes critérios. Para a inteligência existencial, o próprio Gardner, identificou que respondia a seis critérios. Vou apontar cada um destes critérios e discutir seus méritos em relação aos enteógenos.

Um critério aplicável à inteligência existencial é a identificação de um substrato neural a que essa inteligência possa se correlacionar. Gardner (1999a) observou que evidência neuropsicológica recente apoiava a hipótese de que o lobo temporal do cérebro humano abrigava um papel chave na produção de estados místicos e de consciência espiritual (p. 124-125; LaPlante, 1993, Newberg, D'Aquili \& Rause, 2001). Ele também reconheceu que "certos centros cerebrais e neurotransmissores são mobilizados em estados de (consciência alterada), seja pela indução a partir da ingestão de substâncias ou pelo controle da vontade" (Gardner, 1999a, p.125). Outra possibilidade, que Gardner não explora, é que a dimetiltriptamina (DMT) endógena nos humanos pode ter um papel significativo na produção de estados de consciência alterada espontânea ou induzida (Pert, 2001). DMT é uma poderosa substância enteogênica que existe naturalmente no cérebro dos mamíferos (Barker, Monti \& Christian, 1981), sendo, ao mesmo tempo um constituinte da ayahuasca e do rapé angico amazônicos (Ott, 1994). Além disso, a DMT é um análogo próximo do neurotransmissor 5-hidroxitriptamina, ou serotonina. Sabe-se há décadas que a ação neurofarmacológica primária dos psicodélicos se faz sobre os sistemas da serotonina, e a serotonina, sabe-se hoje, está associada aos modos de consciência saudáveis.

Strassman (2001), um pesquisador em psiquiatria, formulou recentemente a hipótese de que a DMT endógena estimula a glândula pineal para criar estados psicodélicos espontâneos como a experiência de proximidade da morte. Esteja ele correto ou não, o 
papel da DMT no cérebro é uma área de pesquisa empírica que merece muito mais atenção, especialmente enquanto pode contribuir para fundamentar com evidências a inteligência existencial.

Outro critério para determinar uma inteligência é a existência de indivíduos com habilidade excepcional dentro do domínio desta inteligência. Infelizmente, a precocidade existencial não é algo suficientemente valorizado na moderna cultura ocidental ao ponto em que especialistas neste domínio sejam celebrados comumente, hoje. Gardner (1999a) observou que dentro do Budismo tibetano a escolha dos lamas envolve a detecção de uma predisposição ao intelecto existencial (se não é pela identificação da reencarnação de um antigo lama, como os budistas tibetanos acreditam) (p. 124). Gardner também cita a consideração de Csikszentmilhalyi sobre "o interesse muito precoce por questões cosmológicas do tipo das relatadas na infância de futuros lideres religiosos como Gandhi e vários futuros físicos inventivos" (Gardner, 1999a, p.124; Csikszentmilhalyi, 1996). Presumivelmente, alguns indivíduos que são convidados a entrar para um mosteiro ou um convento em idade muito precoce, são escolhidos graças a manifestações especiais de consciência existencial. Da mesma forma, nas culturas indígenas, indivíduos que são chamados para as práticas xamânicas - "tem habilidades excepcionais para sonhar, imaginar e entrar em estados de transe" (Larsen, 1976, p.9) - frequentemente demonstram um interesse significativo em questões cosmológicas com muito pouca idade, o que pode ser pensado como uma capacidade especial no domínio da inteligência existencial (Eliade, 1964; Greeley, 1974; Halifax, 1979). ${ }^{7}$

O terceiro critério de Gardner para determinar uma inteligência é um leque identificável das principais habilidades operacionais que manifestam esta inteligência. Gardner (1999a) considerava isto relativamente simples e definiu as principais operações para a inteligência existencial como: 
a capacidade de se localizar em relação aos mais distantes limites do cosmos - o infinito não menos que o infinitesimal - e a capacidade de se localizar com respeito aos aspectos mais existenciais da condição humana: o significado da vida, o sentido da morte, o destino final dos mundos físico e psicológico, algumas experiências profundas como o amor por outro ser humano ou a total imersão numa obra de arte (p.123)

Gardner observou que, assim como em outras mais reconhecidas formas de inteligência, não há nenhuma verdade específica que deva ser alcançada com a inteligência existencial - por exemplo, como a inteligência musical não tem que manifestar-se em qualquer gênero especifico ou categoria musical, a inteligência existencial também não privilegia qualquer sistema filosófico ou doutrina espiritual. Como Gardner (1999a) coloca, "existe (com a inteligência existencial) um potencial - ou capacidade - da espécie de envolver-se em questões transcendentais que pode ser despertada em determinadas circunstâncias" (p.123). Relatos sobre o uso de psicodélicos por ocidentais nos anos 50 e primeiros anos 60 do século passado - produzidos, anteriormente a sua proibição, e alguns podem dizer profanação - revelam o tema recorrente de experiências místicas espontâneas que são consistentes com a capacidade ampliada da inteligência existencial (Huxley, 1954/1971; Masters \& Houston, 1966; Pahnke, 1970; Smith, 1964; Watts, 1958/1969).

Outro critério para o reconhecimento de uma inteligência é identificar-se um desenvolvimento histórico e um conjunto de performances em "estado conclusivo" de especialistas neste campo. Em relação à inteligência existencial, Gardner observa que todas as culturas desenvolveram sistemas espirituais ou metafísicos para lidar com a capacidade humana inerente para questões existenciais, e além disso, que estes sistemas invariavelmente importam em fases ou níveis de sofisticação separando os noviços dos adeptos. Ele usa como ilustração contemporânea deste ponto a descrição do Papa João XXIII de seu treinamento para ascender na hierarquia eclesiástica 
(1999a, p.124). No entanto, a instrução do neófito é parte manifesta de quase todos os treinamentos espirituais, e, novamente, o processo intenso para a incorporação da sabedoria xamânica - incluindo, frequentemente, a utilização apropriada e eficaz de enteógenos - é um excelente exemplo desse processo nas culturas indígenas (Eliade, 1964).

Um quinto critério sugerido por Gardner para determinar uma inteligência é o de sua história evolucionária ou plausibilidade evolucionária. A questão sobre quando e por que a inteligência existencial primeiro surgiu na evolução do gênero Homo é uma questão perene da humanidade. Que ela seja um traço exclusivamente humano é quase axiomático, embora pequeno mas crescente número de pesquisadores estejam dispostos a admitir a possibilidade de formas superiores de cognição entre animais nãohumanos (Masson \& McCarthy, 1995; Vonk, 2003). Gardner (1999a) argumentou que somente no Paleolítico Superior "os seres humanos dentro de uma cultura possuem um cérebro capaz de considerar questões cosmológicas centrais à inteligência existencial" (p.124) e que o desenvolvimento da capacidade de pensamento existencial deve estar correlacionada a "um sentido consciente do espaço finito e do tempo irreversível, dois promissores pontos de partida para estimular explorações imaginárias de esferas transcendentais" ( $p$. 124). Ele também sugeriu que "pensamentos sobre questões existenciais podem ter surgido como respostas a experiências necessárias de dor, talvez como uma maneira de reduzir a dor ou preparar melhor os indivíduos para suportá-la (Gardner, 1999a, p. 125). Assim como para determinar a origem evolucionária da linguagem, traçar uma filogênese da inteligência existencial, é no máximo uma conjectura. Seu papel no desenvolvimento da espécie é igualmente difícil de acessar, embora Winkelman (2000) tenha colocado que a consciência e as práticas xamânicas - e 
presumivelmente também a inteligência existencial - surjam de adaptações psicobiológicas integrando estruturas de desenvolvimento antigas e mais recentes no cérebro hominídeo trino. McKenna (1992) chegou mesmo a postular que a ingestão de substâncias psicoativas tais como os cogumelos enteogênicos pode ter ajudado a estimular desenvolvimentos cognitivos tais como o pensamento existencial e linguístico em nossos ancestrais proto-humanos. Alguns pesquisadores nos anos 50 e 60 detectaram um aumento na criatividade e na capacidade de resolver problemas em sujeitos que ingeriram LSD e outras drogas psicodélicas (Harman, McKim, Morgan, Fadiman \& Stolaroff, 1966; Isumi, 1970; Krippner, 1985; Stafford \& Golightly, 1967), capacidades que teriam sido certamente vantajosas aos nossos ancestrais hominídeos. Tais caminhos de investigação começam de novo a ser trilhados tanto por estudiosos acadêmicos como por psiconautas amadores (Dobkin de Rios \& Janiger, 2003; Spitzer, et al., 1996; MAPS Bulletin, 2000).

O último critério mencionado por Gardner como aplicável à inteligência existencial é a suscetibilidade à codificação em um sistema simbólico. Aqui, também, Gardner admite que há evidência abundante a favor de considerar-se a inteligência existencial como uma inteligência. Em suas palavras, "muitos dos mais importantes e duradouros conjuntos de sistemas simbólicos (p.ex. aqueles encontrados na liturgia católica) representam a cristalização de ideias chave e experiências desenvolvidas no interior de instituições culturais" (1999a, p. 123). Outro exemplo marcante que ilustra esse ponto é o simbolismo mítico atribuído às visões da ayahuasca entre os Tukano, um povo indígena amazônico. Reichel-Dolmatoff (1975) fez um estudo detalhado destas visões pedindo a uma variedade de informantes para desenhar suas representações com galhos no chão (p.174). Ele compilou 20 motivos comuns, observando que a maioria deles tinha uma estranha semelhança com os padrões de fosfeno (i.e. fenômenos visuais percebidos na ausência de estímulos externos ao 
se aplicar uma ligeira pressão sobre o globo ocular) compilados por Knoll (Oster, 1970). Os Tukano interpretam estes fenômenos neuropsicológicos como simbolicamente significativos de acordo com sua mitologia tradicional baseada nas experiências com a ayahuasca, e refletindo a codificação de ideias existenciais de sua cultura.

Narby (1998) também estudou a codificação de símbolos gerados durante experiências com a ayahuasca traçando semelhanças entre os motivos de cobras trançadas nas visões de xamãs amazônicos e a estrutura de dupla hélice do ácido desoxirribonucleico. Ele encontrou semelhanças marcantes entre representações do conhecimento biológico de xamãs indígenas e o dos geneticistas modernos. Mais recentemente, Narby (2002) prosseguiu neste estudo trazendo especialistas em biologia molecular para a Amazônia para participar em cerimônias de ayahuasca com xamãs experientes, uma iniciativa que ele sugere possa trazer cruzamentos úteis em domínios divergentes do conhecimento humano.

Os dois outros critérios determinantes de uma inteligência são a sua comprovação por atividades psicológicas experimentais e por instrumentos psicométricos, e comprovação por pesquisas psicométricas. Gardner (1999a) sugere que a inteligência existencial é mais discutível nesses domínios, citando testes de personalidade que procuram quantificar religiosidade e espiritualidade. Ele observa que "mantém-se pouco claro o que está exatamente sendo sondado com estes instrumentos e se relatos pessoais são um índice confiável nesta sondagem" (p.125). Ao que parece, estados transcendentais de consciência e o conhecimento que eles produzem não se prestam à quantificação ou a replicação em experiência laboratorial. No entanto, Strassman, Qualis, Uhlenhuth e Kellner (1994) desenvolveram um instrumento psicométrico - a Escala de Avaliação Alucinogênica - para medir a resposta humana à administração 
intravenosa do DMT, e este teste vem sendo utilizado de forma confiável para outras experiências psicodélicas (Riba, RodriguezFormells, Strassman \& Barbanoj, 2001).

Uma área de pesquisa psicológica empírica que estimulou ostensivamente 0 que pode ser considerado como inteligência existencial foi a das investigações clínicas com psicodélicos. Até que esta área de pesquisa se tornasse academicamente pouco aceitável, e mesmo politicamente impossível, no início dos anos 70, psicólogos e pesquisadores clínicos exploraram experimentalmente experiências transcendentais induzidas usando drogas, com objetivos tanto de ciência pura como da busca de tratamentos médicos (Abramson, 1967; Cohen, 1964; Grinspoon \& Bakalar, 1979/1998; Masters \& Houston, 1966). Uma das mais famosas entre estas foi a pesquisa da "Sexta-feira Santa" de Pahnke (1970), que buscava induzir experiências espirituais com psilocibina utilizando uma metodologia randômica e de controle duplo-cego. Sua conclusão de que estas experiências místicas eram abordadas e apropriadas de forma confiável, apesar dos problemas metodológicos com o design da pesquisa, foi corroborada por um acompanhamento crítico de longa duração (Doblin, 1991) que levantou questões pertinentes sobre os enteógenos e a inteligência existencial.

Pesquisas como a de Pahnke (1970) apesar de suas promessas, foram prematuramente interrompidas a partir da pressão pública de uma população alarmada com o crescente uso recreativo de drogas, na época. Somente há uma década, o governo dos Estados Unidos deu permissão a pesquisadores para retomar (em pequena escala) as investigações com os psicodélicos (Strassman, 2001; Strassman \& Qualis, 1994). Psicólogos cognitivos têm mostrado interesse pelos enteógenos como a ayahuasca (Shanon, 2002). Apesar da sustentação teórica da inteligência existencial ser ou não estabelecida por meios psicométricos ou atividades psicológicas experimentais, a teoria de Gardner estipula expressamente que não necessariamente 
todos os oito critérios devam ser comprovados para que uma inteligência possa se qualificar. No entanto, Gardner afirmou "considerar o fenômeno desconcertante o bastante, e a distância com as outras inteligências suficientemente grande" (p. 127) para mostrar-se relutante "no momento, em acrescentar a inteligência existencial à (sua) lista... No máximo ele aceita, no estilo Fellini, brincar com a ideia de '8 1/2 inteligências'" (p.127). Eu defendo que as pesquisas com os enteógenos e outros meios de alteração de consciência deverão, adiante, apoiar mais consistentemente a proposta de considerar a inteligência existencial como um domínio cognitivo válido.

\section{Implicações Educacionais}

Retomando e ampliando a discussão de Gardner sobre a inteligência existencial, espero ter fortalecido a questão pela sua inclusão como um domínio cognitivo válido. Fazer isto, no entanto, levanta questões sobre que caminhos a aceitação da inteligência existencial abriria para a teoria e a prática da educação ocidental contemporânea. Como podemos promover essa até agora negligenciada forma de inteligência e permitir que ela seja usada de forma construtiva? Há certamente uma série de práticas educacionais que poderiam ser usadas para estimular a cognição neste domínio, muitas das quais poderiam ser imediatamente implementadas sem muita controvérsia. Ainda assim, eu, intencionalmente, levanto a proposta do uso dos enteógenos neste domínio - não com crianças pequenas, mas talvez com adolescentes na sua passagem para a vida adulta - desafiando assim teóricos, educadores e proponentes de políticas públicas.

Aldous Huxley identificou o potencial dos enteógenos para a educação na cultura ocidental contemporânea. Embora mais conhecido como romancista que como filósofo da educação, Huxley 
dedicou um tempo considerável ao tópico da educação, particularmente na fase final de sua obra. Como em boa parte de sua literatura, as observações e críticas de Huxley às forças sócioculturais dominantes em sua época foram inteligentemente prescientes; elastêm igual ou maior relevância no século XXI do que quando foram escritas. Notavelmente, e isso é relevante para minha tese, Huxley encarava os enteógenos como possíveis instrumentos educacionais.

$\mathrm{Na}$ situação atual, a maioria dos indivíduos perde, no decorrer de sua educação, toda abertura para inspiração, toda capacidade de tornar-se consciente de outras coisas além das enumeradas no catálogo da Sears Roebuck que constitui o convencionado mundo "real".... É esperar demasiado acreditar que um sistema educacional possa algum dia ser concebido buscando resultados, em termos de desenvolvimento humano, comparáveis ao tempo, dinheiro, energia e devoção que Ihe são dedicados? Em um tal sistema educacional, a mescalina ou outras substâncias químicas poderão talvez ter um papel, tornando possível aos jovens "provar e ver" o que eles sempre aprenderam de segunda mão... nos escritos de religiosos ou no trabalho de poetas, pintores e músicos (Carta ao Dr. Humphrey Osmond, 10 de abril de 1953, in Horowitz \& Palmer, 1999, p.30)

Numa expressão mais literária desta noção, o último romance de Huxley (1962), A Ilha, retratava uma cultura ideal que alcançara um equilíbrio entre o pensamento científico e o espiritual, e que incorporara o uso ritualizado de enteógenos na educação. A representação do uso da droga retratada $\mathrm{n}^{\prime} A$ Ilha contrasta fortemente com o mais amplamente conhecido soma de seu romance anterior, Admirável Mundo Novo (1932/1946): o soma era um pacificador que emudecia a curiosidade e servia aos interesses da elite dirigente, enquanto o enteógeno "moksha" d'A IIha permitia experiências liminares entre jovens e adultos, que estimulavam reflexão profunda, auto-realização, e, entendo eu, a inteligência existencial. 
A obra de Huxley aponta para um reconhecimento implícito da capacidade dos enteógenos usados como instrumentos educacionais. O conceito de instrumento refere-se aqui não somente a aparelhos físicos criados para ajudar a produção material, mas seguindo Vygotsky (1978), mais amplamente, a meios de mediação simbólica e/ou cultural entre a mente e o mundo (Cole, 1996; Wertsch, 1991). Evidentemente, obter benefícios educacionais de um instrumento requer muito mais que tê-lo e jogar com ele; é preciso que se tenha um respeito intrínseco pelo objeto enquanto instrumento, um sistema cultural no qual o instrumento seja valorizado como tal, e guie ou ensine os adeptos a utilizá-lo para obter uma direção útil. Como colocou Larsen (1976), discutindo o fenômeno dos intitulados "xamãs" na cultura ocidental, experimentando com as substâncias químicas que produziam alteração de consciência: "não temos nenhum vocabulário simbólico, ou tradição mitológica própria, para tornar essas experiências compreensíveis para nós... nenhum xamã antigo que nos assegure que [nossa experiência xamânica de] desmembramento seja seguida pelo renascimento" (p.81). Dada a história recente destas substâncias na cultura moderna ocidental, é pouco surpreendente que elas tenham sido demonizadas (Hoffmann, 1980). No entanto, as práticas culturais que utilizam tradicionalmente os enteógenos como agentes terapêuticos, incorporam, de forma consistente, salvaguardas de proteção - um espaço e um setting ${ }^{8}$, dosagens estabelecidas, e o respeito mítico-cultural (Zinberg, 1984). O medo que surge inevitavelmente na cultura moderna ocidental ao aproximar-se da questão dos enteógenos tem como origem não propriedades intrínsecas das próprias substâncias, mas, principalmente, uma não-compreensão genérica de seu poder e capacidade como instrumentos. Tanto como uma faca afiada pode ser usada para o bem ou para o mal, dependendo se está nas mãos de 
um cirurgião habilitado ou de um jovem imprudente, também os enteógenos podem ser bem ou mal utilizados.

O uso de um enteógeno como a ayahuasca é um exemplo de uma tradição antiga e ainda viva em muitas culturas de empregar psicoativos como instrumentos que estimulam tipos fundamentais de compreensão (Tupper, 2003). Que estas substâncias sejam capazes de estimular profundamente experiências transcendentes é evidenciado tanto pela literatura acadêmica quanto por relatos anedóticos. Para considerar sua ação, no entanto, é necessário ir além dos esquemas explicativos costumeiros: utilizar a teoria das múltiplas inteligências de Gardner (1999a) como uma grade heurística abre novos caminhos para o entendimento dos enteógenos e seus benefícios potenciais. Ao mesmo tempo, os enteógenos fortalecem a pesquisa proposta por Gardner para uma inteligência existencial. Este artigo busca apresentar estes conceitos de maneira que a possibilidade do uso dos enteógenos possa ser seriamente considerada por aqueles que têm interesse por uma educação nova e transformadora.

\section{NOTAS}

3. A Convenção das Nações Unidas de 1971 sobre substâncias psicotrópicas permite que povos indígenas usem seus medicamentos e sacramentos tradicionais mesmo que estas substâncias sejam proibidas por outras instâncias (Nações Unidas, 1977, Artigo 32.)

4. Santo Daime é o nome tanto do sacramento quanto da religião.

5. Os escritores e aficcionados por drogas William S. Burroughs e Allan Ginsberg (1963) publicaram um relato de sua busca na América do Sul no inicio dos anos 60, mas seu relato é basicamente negativo e não influenciou muitos outros a segui-los. Como etnobotânico Wade Davis observa que sua experiência com o chá "ayahuasca é muitas coisas, menos prazerosa" (2001)

6. Os sete tipos de inteligência originais que Gardner (1983) propôs eram: a linguística, lógico-matemática, espacial, musical, cinestésica, interpessoal e intrapessoal. 
7. Eliade (1964) identifica dois caminhos básicos para se tornar um xamã: (a) a transmissão hereditária, ou tornar-se herdeiro da vocação de uma tradição familiar passada de geração para geração; e (2) a vocação espontânea, ou ser chamado para o xamanismo pelos espíritos. Uma prodigiosa inteligência existencial deve ser manifesta em ambos os casos.

8. O set, ou o espaço, são as expectativas que uma pessoa tem do que a droga fará com ela [sic], considerada no contexto de sua personalidade como um todo. O settingé o ambiente, tanto físico quanto social, no qual a droga é tomada" (Weil, 1972/1986., p.29). Esses fatores influenciam qualquer experiência com drogas psicoativas, mas especialmente os psicodélicos ou enteógenos.

\section{REFERÊNCIAS}

Abramson, H. A. (Ed.). (1967). The use of LSD in psychotherapy and alcoholism. New York: Bobbs-Merrill Co. Ltd.

Adelaars, A. (2001, 21 April). Court case in Holland against the use of ayahuasca by the Dutch Santo Daime Church. Acessado em 10, Maio, 2004

http://www.santodaime.org/community/news/2105 holland.htm

Barker, S. A., Monti, J. A., \& Christian, S. T. (1981). N,NDimethyltryptamine: An endogenous hallucinogen. International Review of Neurobiology. 22, 83-110.

Brown, M. F. (1986). Tsewa's gift: Magic and meaning in an Amazonian society. Washington, DC: Smithsonian Institution Press.

Burroughs, W. S. \& Ginsberg, A. (1963). The yage letters. San Francisco: City Lights Books.

Callaway, J. C., McKenna, D. J., Grob, C. S., Brito, G. S., Raymon, L. P., Poland, R. E., Andrade, E. N., \& Mash, D.C. (1999). Pharmacokinetics of hoasca alkaloids in healthy humans. Journal of Ethnopharmacology, 65, 243-256.

Center for Cognitive Liberty and Ethics. (2003, September 5). 10th Circuit: Church likely to prevail in dispute over hallucinogenic tea. Acessado em 7 Fevereiro, 2004, em http://www.cognitiveliberty.org/dll/udv 10prelim.htm 
Cohen, S. (1964). The beyond within: The LSD story. New York: Atheneum.

Cole, M. (1996). Culture in mind. Cambridge, MA: Harvard University Press.

Csikszentmilhalyi, M. (1996). Creativity. New York: Harper Collins.

Davis, W. (2001, January 23). In Coulter, P. (Producer). The end of the wild [radio program]. Toronto: Canadian Broadcasting Corporation.

Dobkin de Rios, M. (1973). The influence of psychotropic flora and fauna on Maya religion. Current Anthropology, 15(2), 147-164.

Dobkin de Rios, M. (1984). Hallucinogens: Cross-cultural perspectives. Albuquerque, NM: University of New Mexico Press.

Dobkin de Rios, M. (1996). On "human pharmacology of hoasca": A medical anthropology perspective. The Journal of Nervous and Mental Disease, 184(2), 95-98.

Dobkin de Rios, M., \& Janiger, O. (2003). LSD, spirituality, and the creative process. Rochester, VT: Park Street Press.

Doblin, R. (1991). Pahnke's "Good Friday Experiment": A long-term follow-up and methodological critique. The Journal of Transpersonal Psychology, 23(1): 1-28.

Eliade, M. (1964). Shamanism: Archaic techniques of ecstasy (W. R. Trask, Trans.) New York: Pantheon Books.

Eliade, M. (1978). A history of religious ideas: From the stone age to the Eleusinian mysteries (Vol. 1) (W. R. Trask, trans.). Chicago, IL: University of Chicago Press.

Fikes, J. C. (1996). A brief history of the Native American Church. In H. Smith \& R. Snake (Eds.), One nation under god: The triumph of the Native American church (p. 167-173). Santa Fe, NM: Clear Light Publishers.

Gardner, H. (1983). Frames of mind: The theory of multiple intelligences. New York: Basic Books.

Gardner, H. (1999a). Are there additional intelligences? In J. Kane (Ed.), Education, information, transformation: Essays on learning and thinking (pp. 111-131). Upper Saddle River, NJ: Prentice-Hall. 
Gardner, H. (1999b). Intelligence reframed: Multiple intelligences for the 21st century. New York: Basic Books.

Greeley, A. M. (1974). Ecstasy: A way of knowing. Englewood Cliffs, NJ: Prentice- Hall.

Grinspoon, L., \& Bakalar, J. B. (1998). Psychedelic drugs reconsidered. New York: The Lindesmith Center. (Texto original publicado em 1979)

Grob, C. S. (1999). The psychology of ayahuasca. In R. Metzner (Ed.), Ayahuasca: Hallucinogens, consciousness, and the spirit of nature (pp. 214-249). New York: Thunder's Mouth Press.

Grob, C. S., McKenna, D. J., Callaway, J. C., Brito, G. C., Neves, E. S., Oberlander, G., Saide, O. L., Labigalini, E., Tacla, C., Miranda, C. T., Strassman, R. J., \& Boone, K. B. (1996). Human psychopharmacology of hoasca, a plant hallucinogen used in ritual context in Brazil. The Journal of Nervous and Mental Disease, 184(2), 86-94.

Halifax, J. (1979). Shamanic voices: A survey of visionary narratives. New York: Dutton.

Harman, W. W., McKim, R. H., Mogar, R. E., Fadiman, J., \& Stolaroff, M. (1966). Psychedelic agents in creative problem-solving: A pilot study. Psychological Reports, 19: 211-227.

Hofmann, A. (1980). LSD: My problem child. (J. Ott, Trans.). New York: McGraw-Hill.

Horowitz, M., \& Palmer, C. (Eds.). (1999). Moksha: Aldous Huxley's classic writings on psychedelics and the visionary experience. Rochester, VT: Park Street Press.

Huxley, A. (1946). Brave new world: A novel. New York: Harper \& Row. (Original work published 1932)

Huxley, A. (1962). Island. New York: Harper \& Row.

Huxley, A. (1971). The doors of perception \& heaven and hell. Middlesex, UK: Penguin Books. (Texto original publicado em 1954)

Izumi, K. (1970). LSD and architectural design. In B. Aaronson \& H. Osmond, (Eds.), Psychedelics: The uses and implications of hallucinogenic drugs (pp. 381-397). Garden City, NY: Anchor Books. 
John-Steiner, V., \& Souberman, E. (1978). Afterword. In L. Vygotsky, Mind in society: The development of higher psychological processes (pp. 121-133). Cambridge, MA: Harvard University Press.

Julien, R. M. (1998). A primer of drug action: A concise, non-technical guide to the actions, uses, and side effects of psychoactive drugs (8th ed.). Portland, OR: W.H. Freeman \& Company.

Krippner, S. (1985). Psychedelic drugs and creativity. Journal of Psychoactive Drugs, 17(4), 23-245.

LaBarre, W. (1989). The peyote cult (5th ed.). Norman, OK: University of Oklahoma Press.

LaPlante, E. (1993). Seized: Temporal lobe epilepsy as a medical, historical, and artistic phenomenon. New York: Harper-Collins.

Larsen, S. (1976). The shaman's doorway: Opening the mythic imagination to contemporary consciousness. New York: Harper \& Row.

Lewin, L. (1997). Phantastica: A classic survey on the use and abuse of mind-altering plants. Rochester, VT: Park Street Press. (Texto original publicado em 1924)

Luna, L. E. (1984). The concept of plants as teachers among four mestizo shamans of Iquitos, northeastern Peru. Journal of Ethnopharmacology, 11(2), 135-156.

MAPS (Multidisciplinary Association for Psychedelic Studies) Bulletin. (2000). Psychedelics \& Creativity. 10(3). Retrieved February 15, 2004 from: http://www.maps.org/news-letters/v10n3/

Masson, J. M., \& McCarthy, S. (1995). When elephants weep: The emotional lives of animals. New York: Delta Books.

Masters, R. E. L., \& Houston, J. (1966). The varieties of psychedelic experience. New York: Holt, Rinehart and Winston.

McKenna, D. J. (1999). Ayahuasca: An ethnopharmacologic history. In R. Metzner (Ed.), Ayahuasca: Hallucinogens, consciousness, and the spirit of nature (pp. 187-213). New York: Thunder's Mouth Press.

McKenna, D. J., Towers, G. H. N., \& Abbot, F. (1984). Monoamine oxidase inhibitors in South American hallucinogenic plants: Tryptamine and $\square$-carboline constituents of ayahuasca. Journal of Ethnopharmacology, 10(2), 195-223. 
McKenna, T. (1992). Food of the gods: The search for the original tree of knowledge. New York: Bantam.

Metzner, R. (1999). Introduction: Amazonian vine of visions. In R. Metzner (Ed.), Ayahuasca: Hallucinogens, consciousness, and the spirit of nature (pp. 1-45). New York: Thunder's Mouth Press.

Myerhoff, B. G. (1974). Peyote hunt: The sacred journey of the Huichol Indians. Ithaca, NY: Cornell University Press.

Narby, J. (1998). The cosmic serpent: DNA and the origins of knowledge. New York: Jeremy P. Tarcher/Putnam.

Narby, J. (2002). Shamans and scientists. In C.S. Grob (Ed.), Hallucinogens: A reader (pp. 159-163). New York: Jeremy P. Tarcher/Putnam.

Newberg, A., D'Aquili, E., \& Rause, V. (2001). Why god won't go away: Brain science and the biology of belief. New York: Ballantine Books.

Oster, G. (1970). Phosphenes. Scientific American, 222(2), 83-87.

Ott, J. (1994). Ayahuasca analogues: Pangæan entheogens. Kennewick, WA: Natural Products Co.

Pahnke, W. (1970). Drugs and Mysticism. In B. Aaronson \& H. Osmond (Eds.), Psychedelics: The uses and implications of hallucinogenic drugs (pp. 145-165). Garden City, NY: Anchor Books.

Pande, C. G. (1984). Foundations of Indian culture: Spiritual vision and symbolic forms in ancient India. New Delhi: Books \& Books.

Pert, C. (2001, May 26). The matter of emotions. Paper presented at the Remaining Human Forum, University of British Columbia, Vancouver.

Reichel-Dolmatoff, G. (1975). The shaman and the jaguar: A study of narcotic drugs among the Indians of Colombia. Philadelphia: Temple University Press.

Riba, J., Rodriguez-Fornells, A., Urbano, G., Morte, A., Antonijoan, R., Montero, M., Callaway, J. C., \& Barbanoj, M. J. (2001). Subjective effects and tolerability of the South American psychoactive beverage Ayahuasca in healthy volunteers. Psychopharmacology, 154, 85-95. 
Riba, J., Rodriguez-Fornells, A., Strassman, R. J., \& Barbanoj, M. J. (2001). Psychometric assessment of the Hallucinogen Rating Scale in two different populations of hallucinogen users. Drug and Alcohol Dependence, 62(3), 215-223.

Ruck, C., Bigwood, J., Staples, D., Ott, J., \& Wasson, R. G. (1979). Entheogens. The Journal of Psychedelic Drugs, 11(1-2), 145-146.

Santo Daime. (2004). Santo Daime: The rainforest's doctrine. Acessado em 14 Maio, 2004, em http://www.santodaime.org/indexy.htm

Schultes, R. E., \& Hofmann, A. (1992). Plants of the gods: Their sacred, healing, and hallucinogenic powers. Rochester, VT: Healing Arts Press.

Shorter Oxford English Dictionary (3rd ed.). (1973). Oxford, UK: Oxford University Press.

Shanon, B. (2002). The antipodes of the mind: Charting the phenomenology of the ayahuasca experience. Oxford, UK: Oxford University Press.

Smith, H. (1964). Do drugs have religious import? In D. Solomon (Ed.), LSD: The consciousness expanding drug (pp. 155-169). New York: G.P. Putnam's Sons.

Smith, H. (2000). Cleansing the doors of perception: The religious significance of entheogenic plants and chemicals. New York: Tarcher/Putnam.

Smith, H., \& Snake, R. (Eds.). (1996). One nation under god: The triumph of the Native American church. Santa Fe, NM: Clear Light Publishers.

Spitzer, M., Thimm, M., Hermle, L., Holzmann, P., Kovar, K. A., Heimann, H., et al. (1996). Increased activation of indirect semantic associations under psilocybin. Biological Psychiatry, 39(12), 10551057.

Stafford, P., \& Golightly, B. (1967). LSD: The problem-solving psychedelic. New York: Award Books.

Stewart, O. C. (1987). Peyote religion: A history. Norman, OK: University of Oklahoma Press. 
Strassman, R. J. (2001). DMT: The spirit molecule. Rochester, VT: Park Street Press.

Strassman, R. J., \& Qualls, C. R. (1994). Dose-response study of $\mathrm{N}$,Ndimethyltryptamine in humans. I. Neuroendocrine, autonomic and cardiovascular effects. Archives of General Psychiatry, 51(2), 85-97.

Strassman, R. J., Qualls, C. R., Uhlenhuth, E. H., \& Kellner, R. (1994). Dose response study of $\mathrm{N}, \mathrm{N}$-dimethyltryptamine in humans. II. Subjective effects and preliminary results of a new rating scale. Archives of General Psychiatry, 51(2), 98-108.

Tupper, K. W. (2003). Entheogens and education: Exploring the potential of psychoactives as educational tools. Journal of Drug Education and Awareness 1(2), 145-161.

União do Vegetal. (2004). União do Vegetal: Centro espírita beneficente. (Acessado em 14 Maio 2004), in http://www.udv.org.br/english/index.html

United Nations. (1977). Convention on psychotropic substances, 1971. New York: United Nations.

Vonk, J. (2003). Gorilla and orangutan understanding of first- and second-order relations. Animal Cognition, 6(2), 77-86.

Vygotsky, L., (1978). Mind in society: The development of higher psychological processes (M. Cole, V. John-Steiner, S. Scribner, \& E. Souberman, Eds.). Cambridge, MA: Harvard University Press.

Wasson, R. G. (1968). Soma: The divine mushroom of immortality. New York: Harcourt Brace Jovanovich.

Wasson, R. G. (1980). The wondrous mushroom: Mycolatry in Mesoamerica. New York: McGraw-Hill.

Watts, A. (1969). This is it. Toronto: Collier-Macmillan Canada Ltd. (Texto original publicado em 1958)

Weil, A. (1986). The natural mind: A new way of looking at drugs and the higher consciousness. Boston: Houghton Mifflin. (Texto original publicado em 1972).

Wertsch, J. V. (1991). Voices of the mind: A sociocultural approach to mediated action. Cambridge, MA: Harvard University Press. 
Winkelman, M. (2000). Shamanism: The neural ecology of consciousness and healing. Westport, CT: Bergin \& Jarvey.

Zinberg, N. E. (1984). Drug, set, and setting: The basis for controlled intoxicant use. New Haven, CT: Yale University Press. 